PERSONAL ACCOUNT

\section{'Hospital life does have its perks'}

\author{
By Shyam Karia, BDJ Reader Panel, Wales
}

$\mathrm{I}$ $\mathrm{t}$ was in early January that I read my first BBC news article on COVID-19. It referred to this disease as a 'mysterious viral pneumonia' and back then very few of us appreciated its severity. We joked about how it shared the same name as a beer; never did we anticipate the degree of change the world would see.

February came along and I was travelling through Myanmar with four colleagues. We had planned to set up a voluntary medical and dental clinic in a town near Mandalay. The medical team were kept busy throughout; however, as the week progressed our dental clinic started seeing fewer and fewer patients. This is unusual for health camps: locals will routinely travel a few hours from neighbouring villages for treatment. But this time, as Myanmar shares borders with China and Thailand (both reporting confirmed COVID-19 cases), locals didn't fancy the risk of travelling to a big city. This was the first time I took COVID-19 seriously.

Fast forward to April, the UK is under lockdown and people are even drawing parallels between this pandemic and World War II. I am one of four Oral and Maxillofacial DCTs working in a small DGH on the outskirts of London. Since March - with a handful of CWT cases being the exception - all our face-to-face consultations and procedures have been cancelled. Many staff in our hospital have already been redeployed, including our dental nurses, but currently DCTs and other dentally qualified staff are awaiting further instruction. We continue with on-call and clinical commitments (mostly limited to telephone clinics), although there have been many suggestions about possible redeployment locations for us. These include: phlebotomy, assisting OMFS consultants with tracheostomies, and delivering hygiene and comfort on COVID-19 wards.

Hospital life does have its perks. We can leave the house to go to work and we have the opportunity to socialise with colleagues. The routine feels important and there is a definite sense of satisfaction and gratitude at the end of the day. But the hospital can also leave you feeling drained. At work everyone is talking about COVID-19 and when you actually see patients with this disease, reality can be quite daunting. Amazingly though, there is a real team spirit in the hospital which helps keep morale high. Our local community have also donated over $£ 25,000$ worth of gifts. Everything and anything has been considered ranging from chocolates to phone chargers, porridge to protein bars and even a supply of clogs!

Back home, my work has meant I have moved away from living with grandparents. I try to make the most of the sun, spending my days off in the garden or cycling through the countryside. Undoubtedly, the COVID-19 pandemic has made life difficult for everyone, but for me there is solace in recognising that the world has come together and everyone is fighting as one.

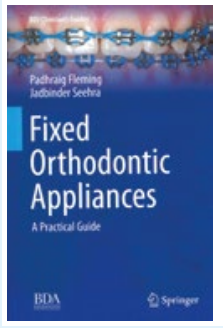

\section{FIXED ORTHODONTIC APPLIANCES: A PRACTICAL GUIDE}

Padhraig Fleming and Jadbinder Seehra;

2019; Springer;

f74.99; pp. 160;

ISBN: 978-3-030-12164-8

The provision of orthodontics has seen significant changes in recent times. With increasing awareness from patients, the use of aligners, and the way treatment is delivered, more recent marketing has been aimed directly at patients, and appliances are being sent directly to patients without a face to face clinical examination. Despite all of this, the majority of comprehensive orthodontic care is still undertaken with fixed appliance systems bonded to teeth. As a result, this book is a timely reminder of the nuances of this vital part of an orthodontist's armamentarium.

This book covers a large scope of fixed orthodontic care, taking the reader through the entire treatment process, doing this in a clear and concise manner. Both authors are widely published academic clinicians with a strong patient facing background, and as such this book strikes a good balance between clinical practice and an evidence base.
The authors describe the complete treatment process involved in the provision of fixed appliance therapy, starting with chapters on appliance choice and instruments used, with a particular focus on the preadjusted edgewise system largely used in the UK. The key stages of orthodontic treatment are then discussed in turn, including alignment, space redistribution, overbite reduction, space closure, and finishing. There is a specific chapter dedicated to the art of wire bending which can sometimes be the most challenging aspect of orthodontic treatment.

I applaud the authors' attempts to include pictures of instruments in use during different treatment protocols, however, demonstrating precise movements with photographs alone is challenging, and I wonder whether future editions could utilise references to appropriate video resources. Also, in the age of adult orthodontics, it may have been useful to add a section within the book regarding bonding appliances to restored surface such as porcelain, amalgam and composite.

Overall, however, this was a well curated book with important information laid out in an easy-to-follow way, and should be essential reading for all those involved in providing fixed orthodontic care for patients. I would highly recommend this book for those undergoing specialist training in orthodontics or orthodontic therapy, as well as a reference book for those providing fixed appliance treatment on a regular basis in their clinical practice. 